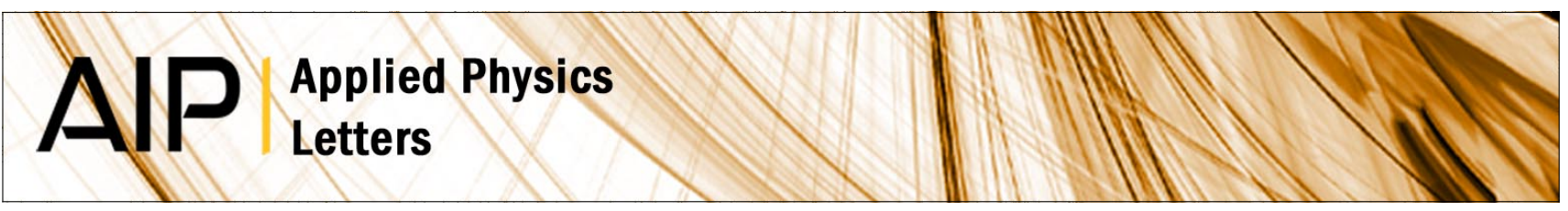

\title{
Surface transfer doping of diamond with a molecular heterojunction
}

D. P. Langley, Y. Smets, C. B. Stark, M. T. Edmonds, A. Tadich et al.

Citation: Appl. Phys. Lett. 100, 032103 (2012); doi: 10.1063/1.3676445

View online: http://dx.doi.org/10.1063/1.3676445

View Table of Contents: http://apl.aip.org/resource/1/APPLAB/v100/i3

Published by the American Institute of Physics.

\section{Related Articles}

Band alignment of vanadium oxide as an interlayer in a hafnium oxide-silicon gate stack structure J. Appl. Phys. 112, 084105 (2012)

Local density of states analysis using Bader decomposition for N2 and CO2 adsorbed on Pt(110)-(1×2) electrodes

J. Chem. Phys. 137, 164705 (2012)

Hard x-ray photoelectron spectroscopy study on band alignment at poly(3,4ethylenedioxythiophene):poly(styrenesulfonate)/ZnO interface

APL: Org. Electron. Photonics 5, 237 (2012)

Fermi-level depinning at the metal-germanium interface by the formation of epitaxial nickel digermanide $\mathrm{NiGe} 2$ using pulsed laser anneal

Appl. Phys. Lett. 101, 172103 (2012)

Hard x-ray photoelectron spectroscopy study on band alignment at poly $(3,4-$ ethylenedioxythiophene):poly(styrenesulfonate)/ZnO interface

Appl. Phys. Lett. 101, 173303 (2012)

\section{Additional information on Appl. Phys. Lett.}

Journal Homepage: http://apl.aip.org/

Journal Information: http://apl.aip.org/about/about_the_journal

Top downloads: http://apl.aip.org/features/most_downloaded

Information for Authors: http://apl.aip.org/authors

\section{ADVERTISEMENT}
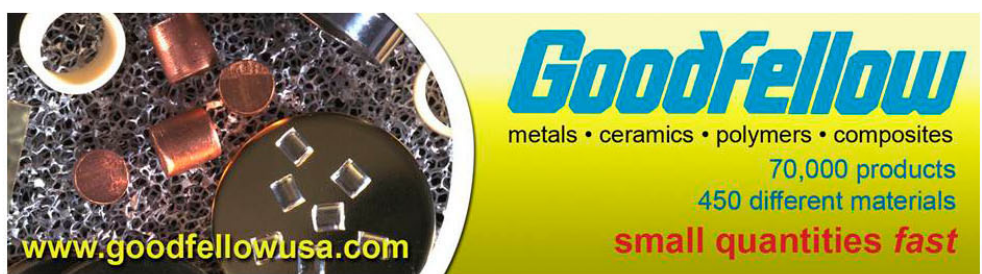


\title{
Surface transfer doping of diamond with a molecular heterojunction
}

\author{
D. P. Langley, ${ }^{1}$ Y. Smets, ${ }^{1}$ C. B. Stark, ${ }^{1}$ M. T. Edmonds, ${ }^{1}$ A. Tadich, ${ }^{2}$ K. J. Rietwyk, ${ }^{1}$ \\ A. Schenk, ${ }^{1}$ M. Wanke, ${ }^{1,3}$ Q.-H. Wu, ${ }^{1}$ P. J. Barnard, ${ }^{4}$ L. Ley, ${ }^{1,3}$ and C. I. Pakes ${ }^{1, a)}$ \\ ${ }^{1}$ Department of Physics, La Trobe University, Victoria 3086, Australia \\ ${ }^{2}$ Australian Synchrotron, 800 Blackburn Road, Clayton, Victoria 3168, Australia \\ ${ }^{3}$ Institut für Technische Physik, Universität Erlangen, Erwin-Rommel-str. 1, 91058 Erlangen, Germany \\ ${ }^{4}$ Department of Chemistry, La Trobe University, Victoria 3086, Australia
}

(Received 20 October 2011; accepted 22 December 2011; published online 17 January 2012)

\begin{abstract}
Surface conductivity and $\mathrm{C} 1 \mathrm{~s}$ core level measurements were employed to show that surface transfer doping of hydrogen-terminated diamond $\mathrm{C}(100)$ can be achieved with a molecular heterojunction formed with $\mathrm{C}_{60} \mathrm{~F}_{48}$ and an intralayer of zinc-tetraphenylporphyrin. Measurement of the shift in the diamond Fermi energy shows that the zinc-tetraphenylporphyrin (ZnTPP) layer modifies the $\mathrm{C}_{60} \mathrm{~F}_{48}$-diamond interaction, modulating the extent of charge transfer between the diamond and the fluorofullerene. In contrast to the case of $\mathrm{C}_{60} \mathrm{~F}_{48}$ acceptors, the presence of a ZnTPP layer prevents the formation of air-induced surface conductivity, showing that the intralayer acts to selectively separate these two doping channels. (C) 2012 American Institute of Physics. [doi:10.1063/1.3676445]
\end{abstract}

Hydrogen termination of diamond results in a negative electron affinity and gives the H-terminated diamond surface an ionisation potential lower than that of any known semiconductor. ${ }^{1}$ This provides a mechanism by which a subsequently adsorbed water layer ${ }^{2}$ or molecular species such as fullerene $\left(\mathrm{C}_{60}\right)$ and fluorofullerene $\left(\mathrm{C}_{60} \mathrm{~F}_{48}\right)^{3}$ may act as a surface acceptor giving rise to $p$-type accumulation of the underlying surface in an otherwise insulating material via surface transfer doping. This is accompanied by an upward band bending at the surface that is equivalent to a downward shift in the Fermi level towards the valence band maximum $(\mathrm{VBM}) .{ }^{4}$ Atmosphere-induced surface conductivity, arising from a redox reaction between an adsorbed water layer and the diamond surface, ${ }^{2}$ has been studied in detail and applied to device applications such as field effect transistors and chemical or biological sensors. ${ }^{5}$ The use of molecular surface acceptors may be an important step towards the fabrication of devices with accurately controllable carrier sheet densities and reproducible characteristics. However, the application of molecular acceptors has been restricted due to the difficulty of preventing atmosphere-induced doping of samples not in vacuum. The present paper addresses this problem.

Unlike conventional semiconductor electronics, where the choice of donor or acceptor is restricted to atomic species that can be incorporated into the crystal lattice of the semiconductor, surface transfer doping offers the possibility of exploiting as acceptors a potentially wide choice of molecular systems with properties that can be systematically modified to control and functionalise the surface properties in addition to being dopants. Functionalisation of the surface charge transfer in diamond to control the level of doping is very much in its infancy and has focused on identifying molecular acceptors of different electron affinity. ${ }^{6}$ An interesting alternative approach which is introduced in this paper is to exploit the interaction of two or more molecular species to

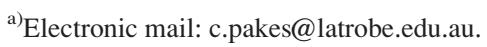

form a "hybrid" acceptor that modifies the extent of surface transfer doping of an underlying $\mathrm{H}$-terminated diamond surface compared to doping with one of the molecules alone. Surface transfer doping of diamond using such a molecular heterojunction has not been reported in the literature, but this approach would be potentially valuable because a diverse range of interesting molecular systems exist with intermolecular interactions that may be functionalised via a response to electrical, magnetic or optical excitation. ${ }^{7}$ This paper explores the effect of the introduction of an intralayer of zinc-tetraphenylporphyrin (ZnTPP) between an acceptor layer and the underlying H-terminated diamond surface. The addition of a ZnTPP layer is found to prevent the development of a surface conductivity upon the subsequent exposure to air but permit fluorofullerene-induced surface conductivity. This importantly shows that a ZnTPP layer may be used to distinguish between the doping channels due to water and fluorofullerene acceptor layers. For the latter case, synchrotron-based photoelectron spectroscopy (PES) is used to show that a strong porphyrin-fluorofullerene interaction in the form of spontaneous charge transfer modulates the extent of doping of the diamond surface compared to the case of $\mathrm{C}_{60} \mathrm{~F}_{48}$ acceptors alone.

Experiments were performed using two synthetic (100)orientated diamond single crystals: an un-doped sample (A) that was utilised for surface conductivity measurements and an identically prepared boron-doped sample (B) used for PES, the bulk conductivity eliminating the effects of charging during photoemission. The samples were cleaned by boiling in acid to remove metallic and non-diamond contamination and hydrogen terminated simultaneously in a microwave H-plasma at a sample temperature of $800^{\circ} \mathrm{C}$ for $45 \mathrm{~min}$. The samples were exposed to air before being transferred to ultra-high vacuum (UHV, $10^{-10} \mathrm{mbar}$ ) at the soft $\mathrm{x}$ ray spectroscopy beamline of the Australian Synchrotron where subsequent processing, conductivity measurements, and PES measurements were performed. The samples were mounted side-by-side onto a Mo sample holder with an 
underlying heater for sample annealing; in this arrangement the treatment of the two samples during annealing and molecular dosing was identical. Prior to dosing with molecular adlayers, the samples were annealed in vacuum for $60 \mathrm{~min}$ at a temperature of $550{ }^{\circ} \mathrm{C}$ to remove air-induced surface conductivity and physisorbed hydrocarbon contamination formed during exposure to ambient conditions. ZnTPP and $\mathrm{C}_{60} \mathrm{~F}_{48}$ adlayers were deposited by sublimation from quartz crucibles at a deposition rate of $1 \AA /$ min determined using a quartz-crystal microbalance. Measurements were performed for the pristine $\mathrm{H}$-terminated diamond surface, after deposition of a ZnTPP layer of thickness 1 monolayer (ML) and following the subsequent stepwise deposition of $\mathrm{C}_{60} \mathrm{~F}_{48}$ up to $0.9 \mathrm{ML}$ in coverage. In addition, conductivity measurements were made after deposition of the ZnTPP layer and subsequent exposure to atmospheric conditions. Conductivity measurements were performed on sample A using a fourpoint-probe system in UHV. PES data from the C1s corelevels were obtained using sample B within the same UHV system using photon energies of 330 and $1200 \mathrm{eV}$, permitting measurements with surface and bulk sensitivity respectively. The binding energy (BE) of all spectra are referenced to the Fermi level using the $4 \mathrm{f}_{7 / 2}$ core level at a BE of $84.00 \mathrm{eV}$ observed for an Au sample in electrical contact with the diamond. Core-level spectra were analysed by background removal via the Shirley technique and peak fitting with a series of Voigt functions as described elsewhere. ${ }^{8}$

Conductivity measurements obtained using sample A for different adsorbate layers are shown in Fig. 1. An atmosphere induced surface conductivity of $2.67 \times 10^{-8} \Omega^{-1}$ was measured prior to annealing the samples in vacuum. After annealing in vacuum at a temperature of $550{ }^{\circ} \mathrm{C}$ a conductivity not exceeding $2 \times 10^{-9} \Omega^{-1}$ was obtained, which was the lower limit of the measurement apparatus. The subsequent deposition of 1 ML ZnTPP did not give rise to an induced surface conductivity. Furthermore, the surface conductivity was found not to recover after the ZnTPP covered surface

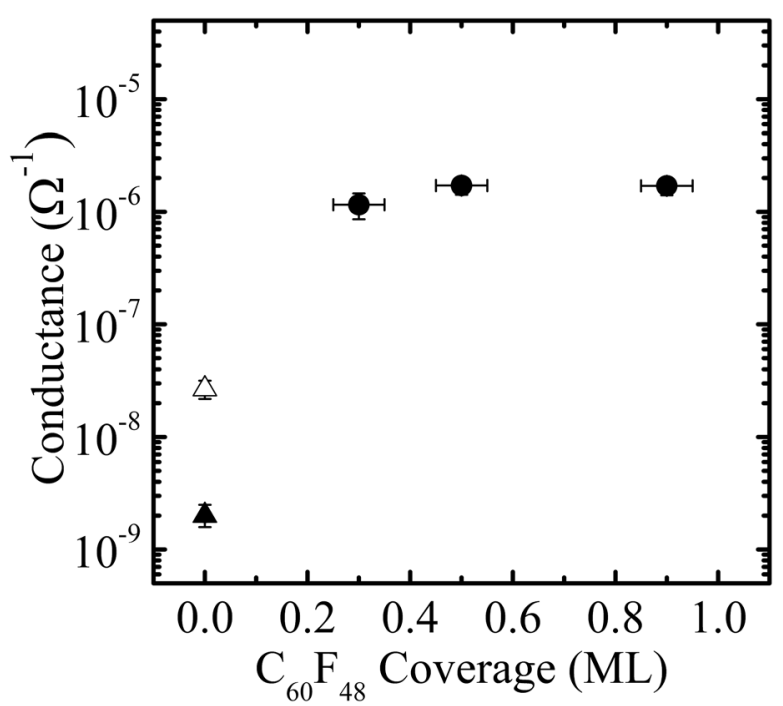

FIG. 1. Induced sheet conductivity as a function of $\mathrm{C}_{60} \mathrm{~F}_{48}$ coverage. Closed circles: fluorofullerene induced surface conductivity with a ZnTPP intralayer of coverage $1 \mathrm{ML}$; open triangle: as prepared sample exhibiting an airinduced surface conductivity with no ZnTPP intralayer; closed triangle: airexposed sample with a ZnTPP intralayer of coverage $1 \mathrm{ML}$. was exposed to air. In contrast, the deposition of $\mathrm{C}_{60} \mathrm{~F}_{48}$ on top of a freshly prepared ZnTPP intralayer caused a significant increase in surface conductivity, saturating at a level of $1.7 \times 10^{-6} \Omega^{-1}$ for a $\mathrm{C}_{60} \mathrm{~F}_{48}$ coverage of $0.5 \mathrm{ML}$. For the case of doping with $\mathrm{C}_{60} \mathrm{~F}_{48}$, PES was used to explore in detail the role of ZnTPP in modifying the doping of the underlying diamond surface. The corresponding PES data obtained for sample B with a photon energy of $330 \mathrm{eV}$ is shown in Fig. 2; the $\mathrm{C}$ 1s core-level spectra are shown for the pristine C(100):H surface (I), after deposition of a ZnTPP intralayer of thickness $1 \mathrm{ML}$ (II) and after subsequent deposition of a $\mathrm{C}_{60} \mathrm{~F}_{48}$ adlayer of thickness 0.3 to $0.9 \mathrm{ML}$ (III-V). For $\hbar \omega=330 \mathrm{eV}$, the escape depth of the detected photoelectrons is approximately $3.5 \AA$, so that the core levels

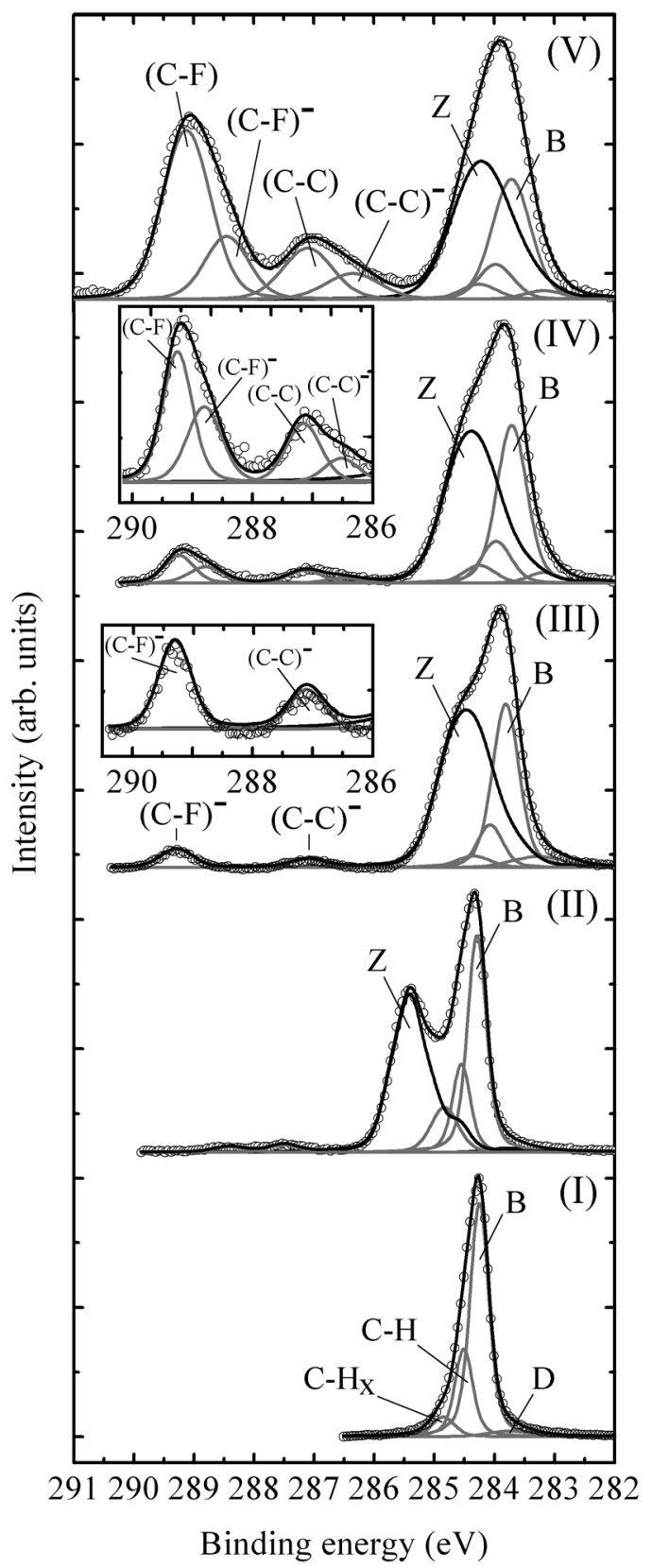

FIG. 2. C1s core level spectra obtained for $\hbar \omega=330 \mathrm{eV}$ : diamond surface after annealing to $550^{\circ} \mathrm{C}$ (I); after introduction of a ZnTPP intralayer of $1 \mathrm{ML}$ coverage (II); after the subsequent introduction of a $\mathrm{C}_{60} \mathrm{~F}_{48}$ adlayer of coverage 0.3 ML (III), 0.5 ML (IV) and 0.9 ML (V). The insets show the fluorofullerene peaks with $\times 5$ magnification. 
corresponding to the adsorbed ZnTPP and $\mathrm{C}_{60} \mathrm{~F}_{48}$ layers are easily identified. For the pristine C(100):H surface, a group of $\mathrm{C} 1 \mathrm{~s}$ lines arise from a dominant core level associated with pure bulk diamond (labelled B) at a $\mathrm{BE}$ of $284.25 \mathrm{eV}$ and surface $\mathrm{C}-\mathrm{H}$ and $\mathrm{C}-\mathrm{H}_{\mathrm{x}}$ components shifted to higher BE by 0.26 and $0.50 \mathrm{eV}$ with respect to the diamond bulk component. In addition, a small surface dimer peak (labelled D) is evident shifted to lower BE. ${ }^{8}$ The ZnTPP layer introduces a broad peak (labelled $\mathrm{Z}$ ) positioned to the high BE side of the diamond peak and two small shake-up satellite peaks. The main ZnTPP peak has been fitted with a series of four $\mathrm{C} 1 \mathrm{~s}$ lines each of which corresponds to differently bonded carbon atoms within the tetraphenylporphyrin molecule; the relative intensity and position of the individual lines is consistent with the work of Cudia et al. ${ }^{9}$ The introduction of ZnTPP causes no apparent shift in the position of the diamond lines relative to $\mathrm{E}_{\mathrm{F}}$ and hence no band bending. This is consistent with the absence of surface conductivity when the porphyrin layer is introduced. The subsequent deposition of fluorofullerene introduces two further $\mathrm{C} 1 \mathrm{~s}$ lines separated by $2.07 \mathrm{eV} \pm 0.05 \mathrm{eV}$ and positioned to the high $\mathrm{BE}$ side of the tetraphenylporphyrin feature. They correspond to the $s p^{2}$ hybridised carbon of the $\mathrm{C}_{60}$ cage with bonds to three neighbouring carbon atoms $(\mathrm{C}-\mathrm{C})$ and to $\mathrm{C}_{60} \mathrm{~F}_{48}$ carbon atoms bonded to fluorine $(\mathrm{C}-\mathrm{F}) .{ }^{10}$ For coverages of $0.5 \mathrm{ML}$ and above the $\mathrm{C}-\mathrm{C}$ and $\mathrm{C}-\mathrm{F}$ lines require two distinct components for a proper fit (labelled C-C, (C-C) ${ }^{-}$and $\mathrm{C}-\mathrm{F},(\mathrm{C}-\mathrm{F})^{-}$, respectively). This indicates the presence of two distinct molecular charge states, the origin of which has been discussed by us elsewhere for the case of $\mathrm{C}_{60} \mathrm{~F}_{48}$ adsorbed directly on the $\mathrm{C}(100): \mathrm{H}$ surface. ${ }^{11}$ The lines at lower BE (labelled $(\mathrm{C}-\mathrm{C})^{-}$and $\left.(\mathrm{C}-\mathrm{F})^{-}\right)$correspond to ionized molecules that participate in surface charge transfer with the underlying layers by accepting an electron, while the lines at higher BE (C-C and C-F) correspond to neutral molecules that do not. An increasing contribution of non-doping neutral fluorofullerenes is observed as the coverage increases and the surface charge transfer process saturates. The $\mathrm{C}_{60} \mathrm{~F}_{48}$ adlayer induces a significant shift of about $1.2 \mathrm{eV}$ in the position of the ZnTPP peak (Z), which signals charging of the ZnTPP, consistent with $p$-type doping of porphyrin-based semiconducting layers by fullerene reported elsewhere. ${ }^{12}$ The additional fluorofullerene causes the diamond bulk peak (B) to shift towards lower $\mathrm{BE}$ as well, signalling $p$-type doping also of the underlying diamond substrate. Changes in the $\mathrm{C} 1 \mathrm{~s}$ core level of diamond are more clearly distinguished in PES measurements with a photon energy $1200 \mathrm{eV}$, where the enhanced escape depth of about $20 \AA$ suppresses the interfering spectral features of the adlayer species. We therefore use data obtained at $1200 \mathrm{eV}$ to accurately determine the downward shifts in the $\mathrm{C} 1 \mathrm{~s}$ core level $\mathrm{BE}$ of diamond relative to $\mathrm{E}_{\mathrm{F}}$. The corresponding downward shift in the diamond Fermi energy with respect to the VBM is plotted in Fig. 3. They were calculated using the fixed energy separation of the VBM to the $\mathrm{C} 1 \mathrm{~s}$ core level of $283.9 \pm 0.1 \mathrm{eV}$ as reported by Maier et al. ${ }^{13}$ In evaluating changes in surface potential from shifts in the $\mathrm{C} 1 \mathrm{~s} \mathrm{BE}$, one has to recognise that band bending may occur on a length scale comparable to the photoelectron escape depth. A convolution of the $\mathrm{C} 1 \mathrm{~s}$ line with the band profile leads to an asymmetric broadening of the diamond

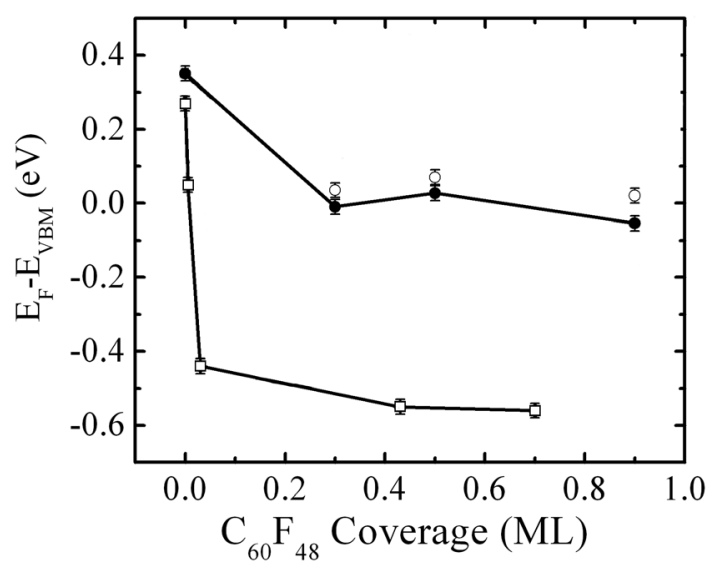

FIG. 3. $E_{\mathrm{F}}-E_{\mathrm{VBM}}$ as a function of $\mathrm{C}_{60} \mathrm{~F}_{48}$ coverage derived from $\mathrm{C} 1$ s core level spectra obtained for $\hbar \omega=1200 \mathrm{eV}$ : open circles before and closed circles after $\mathrm{C} 1 \mathrm{~s} \mathrm{BE}$ corrections. For comparison, $E_{\mathrm{F}}-E_{\mathrm{VBM}}$ values determined for $\hbar \omega=1486.6 \mathrm{eV}$ as a function of $\mathrm{C}_{60} \mathrm{~F}_{48}$ coverage in the absence of a ZnTPP intralayer are shown (open squares from Ref. 14).

$\mathrm{C} 1 \mathrm{~s}$ line towards lower $\mathrm{BE}$ and an underestimation of the surface potential change as derived directly from the core level shift. The necessary correction was applied as explained by Edmonds et al. ${ }^{4,14}$ In Fig. 3 the un-corrected values for $E_{\mathrm{F}}-E_{\mathrm{VBM}}$ are plotted along with the corrected values. The maximum band bending amounts to $0.4 \mathrm{eV}$, with the Fermi energy lying $0.05 \mathrm{eV}$ below the VBM. For comparison corrected $E_{\mathrm{F}}-E_{\mathrm{VBM}}$ values determined as a function of $\mathrm{C}_{60} \mathrm{~F}_{48}$ coverage in the absence of a ZnTPP intralayer are also shown. ${ }^{14}$

The absence of a ZnTPP induced surface conductivity prior to the deposition of fluorofullerene is understood on the basis of its low electron affinity. The electron affinity of ZnTPP is $2.3 \mathrm{eV},{ }^{15}$ similar to that of $\mathrm{C}_{60}$ for which charge transfer from an underlying $\mathrm{H}$-terminated diamond surface proceeds only when sufficient $\mathrm{C}_{60}$ is deposited to form a van-der-Waals solid with increased affinity. ${ }^{3}$ In contrast, $\mathrm{C}_{60} \mathrm{~F}_{48}$ has a high doping efficiency even in its molecular form because the electron affinity increases with fluorination and reaches $4.06 \mathrm{eV}$ for $\mathrm{C}_{60} \mathrm{~F}_{48}{ }^{16}$ The present study shows that $\mathrm{C}_{60} \mathrm{~F}_{48}$ is an efficient surface acceptor even after the introduction of an intermediate ZnTPP layer and that the tetraphenylporphyrin layer modulates the level of doping as indicated by a smaller shift in the diamond Fermi energy compared to the case of $\mathrm{C}_{60} \mathrm{~F}_{48}$ alone. There are two possible roles that the ZnTPP layer may play in modifying the fluorofullerene-surface interaction. The first is as a dielectric layer that displaces the $\mathrm{C}_{60} \mathrm{~F}_{48}$ away from the surface by a distance equal to the thickness of the ZnTPP film but is otherwise inert. As we shall explain below, this scenario is unlikely. Alternatively, the porphyrin intralayer may interact electronically with the fluorofullerene layer. The interaction between porphyrin- and fullerene-derived molecules has been reported extensively in the literature as a potential donor-acceptor heterojunction for organic electronics and photovoltaic applications. ${ }^{12}$ The large downward shift of the $\mathrm{C} 1 \mathrm{~s} \mathrm{BE}$ of ZnTPP in response to the deposition of $\mathrm{C}_{60} \mathrm{~F}_{48}$ (Fig. 2) suggests a significant spontaneous transfer of electrons from $\mathrm{ZnTPP}$ to $\mathrm{C}_{60} \mathrm{~F}_{48}$, leaving the porphyrin film $p$-type doped. Let us determine the relative contribution of 
the holes in ZnTPP and in diamond. A fluorofullerene coverage of $1 \mathrm{ML}$ corresponds to an areal density of $7.8 \times 10^{13}$ $\mathrm{C}_{60} \mathrm{~F}_{48}$ molecules $/ \mathrm{cm}^{-2}$. For a fluorofullerene coverage of 0.3 ML the diamond Fermi energy is aligned with the VBM (Fig. 3). Making use of the universal relationship between $E_{\mathrm{F}}-E_{\mathrm{VBM}}$ for diamond and the underlying hole sheet density, which is independent of acceptor species, ${ }^{4,11,14}$ this infers a hole sheet density of $7 \times 10^{11} \mathrm{~cm}^{-2}$ in the underlying diamond surface. In Fig. 2 (III) the fluorofullerene components are fitted with single $\mathrm{C} 1 \mathrm{~s}$ lines, suggesting a contribution from doping $\mathrm{C}_{60} \mathrm{~F}_{48}$ only. For a coverage of $0.3 \mathrm{ML}$ this corresponds to $2.3 \times 10^{13} \mathrm{~cm}^{-2}$ ionised $\mathrm{C}_{60} \mathrm{~F}_{48}$ molecules, suggesting that about thirty holes are induced in the ZnTPP adlayer for each hole in the underlying diamond surface. This charge transfer couples the two molecules to effectively form a hybrid surface acceptor layer with a doping efficacy lower than that of $\mathrm{C}_{60} \mathrm{~F}_{48}$ alone. For the case of $\mathrm{C}_{60} \mathrm{~F}_{48}$ on the pristine $\mathrm{C}(100): \mathrm{H}$ surface, we have shown that surface charge transfer establishes an interface dipole layer that effectively increases the acceptor energy. ${ }^{4,11}$ For this reason, in the present case an interface dipole arising from the observed spontaneous charge transfer between the ZnTPP intralayer and $\mathrm{C}_{60} \mathrm{~F}_{48}$ would limit the amount of charge that may transfer between the underlying diamond surface and the fluorofullerene, so that the hybrid acceptor layer has a lower doping efficiency than for $\mathrm{C}_{60} \mathrm{~F}_{48}$ alone.

In summary, a combination of surface conductivity and core level $\mathrm{C} 1 \mathrm{~s}$ measurements have been employed to demonstrate the surface transfer doping of $\mathrm{C}(100): \mathrm{H}$ with $\mathrm{C}_{60} \mathrm{~F}_{48}$ coupled to a ZnTPP intralayer. In contrast to doping by $\mathrm{C}_{60} \mathrm{~F}_{48}$ alone, a ZnTPP intralayer was found to prevent atmosphere-induced surface conductivity. The absence of an induced surface conductivity in this case was verified using two additional samples and it was found that the conductivity did not recover under atmospheric conditions for several days. The use of a ZnTPP layer to prevent air-induced conductivity and permit doping with $\mathrm{C}_{60} \mathrm{~F}_{48}$ importantly shows that a ZnTPP intralayer may be used to selectively separate the doping channels of two different acceptor layers. Demonstration of diamond transfer doping with a molecular heterojunction will motivate the study of other functional molecular complexes. In particular, a diverse range of porphyrin-fullerene derivatives exist that are optically active and may permit the optical control of the underlying surface conductivity.

We acknowledge financial support of the Australian Research Council.

${ }^{1}$ J. B. Cui, J. Ristein, and L. Ley, Phys. Rev. Lett. 81, 429 (1998).

${ }^{2}$ F. Maier, M. Riedel, B. Mantel, J. Ristein, and L. Ley, Phys. Rev. Lett. 85, 3472 (2000)

${ }^{3}$ P. Strobel, M. Riedel, J. Ristein, and L. Ley, Nature 430, 439 (2004); P. Strobel, M. Riedel, J. Ristein, L. Ley, and O. Boltalina, Diamond Relat. Mater. 14, 451 (2005).

${ }^{4}$ M. T. Edmonds, C. I. Pakes, A. Tadich, S. Mammadov, W. Zhang, J. Ristein, and L. Ley, Appl. Phys. Lett. 98, 102101 (2011).

${ }^{5}$ CVD Diamond for Electronic Devices, edited by R. S. Sussmann (John Wiley \& Sons, Chichester, 2009).

${ }^{6}$ D. Qi, W. Chen, X. Gao, L. Wang, S. Chen, K. P. Loh, and A. T. S. Wee, J. Am. Chem. Soc. 21, 410 (2011).

${ }^{7}$ T. Nojiri, A. Watanabe, and O. Ito, J. Phys. Chem. A 102, 5215 (1998).

${ }^{8}$ R. Graupner, F. Maier, J. Ristein, L. Ley, and Ch. Jung, Phys. Rev. B 57, 12397 (1998).

${ }^{9}$ C. C. Cudia, P. Vilmercati, R. Larciprete, C. Cepek, G. Zampieri, L. Sangaletti, S. Pagliara, A. Verdini, A. Cossaro, L. Floreano et al., Surf. Sci. 600, 4013 (2006).

${ }^{10}$ P. J. Benning, T. R. Ohno, J. H. Weaver, P. Mukherjee, J. L. Adcock, R. N. Compton, and B. I. Dunlap, Phys. Rev. B 47, 1589 (1993).

${ }^{11}$ M. T. Edmonds, M. Wanke, A. Tadich, H. M. Vulling, K. J. Rietwyk, P. L. Sharp, C. B. Stark, Y. Smets, A. Schenk, Q.-H. Wu, L. Ley, and C. I. Pakes, "Surface transfer doping of hydrogen-terminated diamond by C60F48: energy level scheme and doping efficiency", J. Chem. Phys. (submitted).

${ }^{12}$ P. Vilmercati, C. Cudia, R. Gebauer, P. Ghosh, S. Lizzit, L. Petaccia, C. Cepek, R. Larciprete, A. Verdini, L. Floreano et al., J. Am. Chem. Soc. 131, 644 (2009).

${ }^{13}$ F. Maier, J. Ristein, and L. Ley, Phys. Rev. B 64, 165411 (2001).

${ }^{14}$ M. T. Edmonds, C. I. Pakes, A. Tadich, S. Mammadov, W. Zhang, J. Ristein, and L. Ley, Phys. Stat. Solidi (a) 208, 2062 (2011).

${ }^{15}$ M.-S. Liao and S. Scheiner, J. Chem. Phys. 117, 205 (2002).

${ }^{16}$ R. Mitsumoto, T. Araki, E. Ito, Y. Ouchi, K. Seki, K. Kikuchi, Y. Achiba, H. Kurosaki, T. Sonoda, H. Kobayashi et al., J. Phys. Chem. A 102, 552 (1998). 